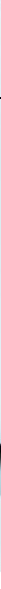

\title{
Warum Ausschreibungen die Versorgung mit Zytostatika gefährden
}

Am 12.10.2016 hat das Bundeskabinett den Entwurf eines „Gesetzes zur Stärkung der Arzneimittelversorgung in der GKV“ beschlossen. Darin heißt es unter anderem: „Mit diesem Gesetz wird [...] die Möglichkeit der Krankenkassen, die Versorgung mit individuell in Apotheken hergestellten parenteralen Zubereitungen aus Fertigarzneimitteln in der Onkologie zur unmittelbaren ärztlichen Anwendung bei Patienten auch durch Verträge mit Apotheken sicherzustellen, gestrichen “. Das heißt: Tritt das Gesetz in Kraft, können Krankenkassen zukünftig nicht mehr festlegen, in welcher Vertragsapotheke Zytostatika zubereitet werden. Dies begrüßen wir ausdrücklich. Denn: Neben den zum Teil großen Entfernungen zwischen Vertragsapotheke und Arztpraxis sprechen aus unserer Sicht noch weitere Gründe für die mit der Gesetzesnovelle verbundene freie Apothekenwahl:

Zytostatika-Zubereitungen sind individuelle Erzeugnisse. Sie sind kein standardisiertes Produkt, keine fertige Ware. Die Zubereitung von Zytostatika ist ein komplexer Prozess, an dem Patient, Arzt und Apotheker beteiligt sind. Dieser Prozess beginnt mit der Krebsdiagnose des Patienten, gefolgt vom zunehmend individuelleren Therapiekonzept, der Therapie- und Terminplanung, der Kommunikation mit der zubereitenden Apotheke, der Zusendung des Therapieplanes, der Rezeptausstellung, der planbaren Rezeptierung oder der Ad-hoc-Bestellung, der Anlieferung der Zytostatika, der Kontrolle der Lieferung, der erneuten Testung des Patienten auf Therapiefähigkeit, der erneuten Zuordnung von Patient und Zytostatika sowie der endgültigen Verabreichung der Mittel.

In der sicheren Applikation der Zytostatika steht der Onkologe nicht nur in der Verantwortung für seine Patienten, sondern auch für seine damit befassten Mitarbeiter. Gefahren für die tägliche Arbeit mit den Substanzen (Mutagenität, Teratogenität) sind nur vermeidbar, wenn alle Abläufe zusammen mit einer Apotheke präzise geplant und standardisiert werden und seitens des Arztes eine genaue Risikokontrolle möglich ist.

Dieser komplexe Prozess muss für den Onkologen in Kooperation mit dem Apotheker unter Wahrung des Patientenrechtes zur freien Wahl der Apotheke vollständig transparent und frei organisierbar sein. Mit einer Ausschreibung dringen die Kassen also nicht nur in die Entscheidungskompetenz des Arztes ein, sondern letztlich auch in das besondere geschützte Vertrauensverhältnis von Arzt und Patient.

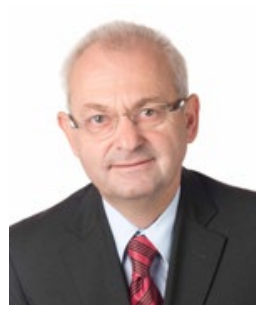

Prof. Dr. Stephan Schmitz

Vorsitzender des Berufsverbandes der Niedergelassenen Hämatologen und Onkologen in Deutschland e. V., Köln

Dort, wo die Qualität einer Leistung nicht beurteilt werden kann, darf es keine Ausschreibung nur zum niedrigsten Preis geben. Die Qualität der Versorgung von Krebskranken würde sich massiv verschlechtern.

Genauso wie wir uns dagegen wehren, dass gesetzliche Krankenkassen die Ausschreibungen zu Lasten der Versorgungsqualität der Patienten durchführen, wollen wir auch kein Oligopol mit wenigen zubereitenden Apotheken. Durch die Ausschreibung fördern die Krankenkassen Oligopole. Wir niedergelassen Hämatologen und Onkologen brauchen insbesondere in $\mathrm{Zu}$ kunft unter Berücksichtigung des rasanten Fortschritts bei den onkologischen Medikamenten Zytostatika-Apotheker/innen mit ihrer besonderen klinisch-pharmakologischen Kompetenz und auch ihren individuellen Beratungsleistungen. Diese Versorgung sollte möglichst breit regional verfügbar bleiben.

Aus Sicht der einzelnen onkologischen Praxis ist die Ausschreibung ein Vertrag zulasten Dritter. Mehrere Ausschreibungen würden den Mehraufwand für die Praxis enorm erhöhen. Der personelle Mehraufwand bei nur einer Ausschreibung ist bereits ein Zusatzaufwand. Darüber hinaus führen die zusätzlichen Schnittstellen in der Regel zu einer reduzierten Prozessund schlechteren Versorgungsqualität. Der Zusatzaufwand der Praxen wird nicht honoriert.

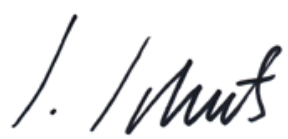

